\title{
Nanofabrication route to achieve sustainable production of next generation defect-free graphene: analysis and characterisation
}

https://doi.org/10.1515/nanofab-2020-0101

Received Oct 11, 2021; accepted Nov 04, 2021

\begin{abstract}
In the past two decades, graphene has been one of the most studied materials due to its exceptional properties. The scalable route to cost-effective manufacture defect-free graphene has continued to remain a technical challenge. Intrinsically defect-free graphene changes its properties dramatically, and it is a challenging task to control the defects in graphene production using scaled-down subtractive manufacturing techniques. In this work, the exfoliation of graphite was investigated as a sustainable low-cost graphene manufacturing technique. The study made use of a simple domestic appliance e.g., a kitchen blender to churn graphene in wet conditions by mixing with N-Methyl2-pyrrolidone (NMP). It was found that the centrifugal forceinduced turbulent flow caused by the rotating blades exfoliates graphite flakes to form graphene. The technique is endowed with a high yield of defect-free graphene $(0.3 \mathrm{~g} / \mathrm{h})$ and was deemed suitable to remove $10 \%$ fluoride content from the water and color absorption from fizzy drinks.
\end{abstract}

Keywords: Graphite exfoliation; Graphene; Kitchen blender

Corresponding Author: Krishanu Biswas: Department of Materials Science and Engineering, Indian Institute of Technology Kanpur, Kanpur, 208016, India; Email: kbiswas@iitk.ac.in

Shikhar Misra: Department of Materials Science and Engineering, Indian Institute of Technology Kanpur, Kanpur, 208016, India Nirmal Kumar Katiyar: Department of Materials Science and Engineering, Indian Institute of Technology Kanpur, Kanpur, 208016, India; School of Engineering, London South Bank University, SE10AA, London, United Kingdom of Great Britain and Northern Ireland Arvind Kumar: Department of Mechanical Engineering, Indian institute of Technology Kanpur, Kanpur, 208016, India

Saurav Goel: School of Engineering, London South Bank University, SE10AA, London, United Kingdom of Great Britain and Northern Ireland; Department of Mechanical Engineering, Indian Institute of Technology Guwahati, Guwahati, 781039, India; Department of Mechanical

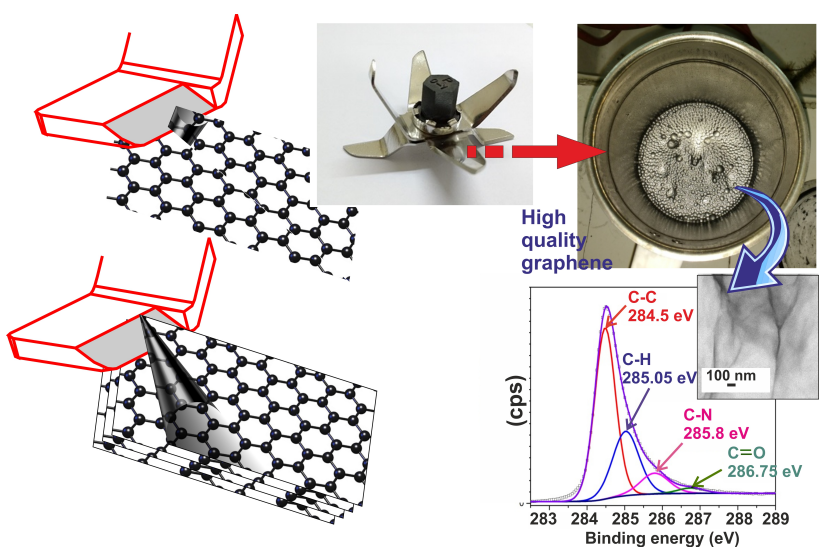

Graphical abstract

\section{Introduction}

Graphene, a $21^{\text {st }}$ Century 'wonder' material is being used for a wide range of engineering applications such as selfhealing, sensors, drug delivery, water filtration and many other newly emerging areas [1-3]. Graphene as being a oneatom-thick layer of carbon has changed the technological landscape of materials development by combining with other materials [4-7]. This has consequently led to evolving methods for graphene manufacturing. The scientific community has discovered many methods for graphene preparation, such as mechanical exfoliation, chemical exfoliation and deposition techniques such as the chemical vapour deposition. A common goal thus far with respect to these developments has been to focus on achieving defect-free graphene for its use in novel applications such as sensing, catalysis, nanocomposites [8-13]. The ambition to achieve high-yield from these methods has not been fulfilled as yet and a robust and cost-effective method to prepare graphene with high yield has continued to remain a challenge. The mechanical exfoliation of graphite to prepare graphene on

Engineering, University of Petroleum and Energy Studies, Dehradun, 248007, India 
a large scale is a widely studied method. However, controlling the synthesis induced defects in the graphene or obtaining defect-free graphene via this technique has continued to be a challenge. Normally, the synthesis of graphene consists of two distinct strategies. The first one involves transforming any carbonaceous material containing layers of graphene by physical or chemical exfoliation methods. This may include exfoliation of graphite [14], unzipping of carbon nanotube [15], boiling of graphite with a strong oxidizing agent followed by its reduction [16, 17], etc.

The other strategy involves the growth of graphene from some carbonaceous precursors. This can be realized by solvothermal reaction [18], arc discharge [19] and chemical vapour deposition [20]. However, besides being expensive, none of these methods can be used for large scale production of graphene. Producing graphene using liquidphase exfoliation is reported to provide large-scale, cheap, and defect-free graphene [14]. In addition, graphene is produced from graphite using microfluidizers [21]. Compared to the oxidation exfoliation of graphite, the liquid phase exfoliation method proves to be both cheaper and produce better quality graphene as has been observed by the Raman spectroscopy. The oxidation exfoliation method can produce large quantities of graphene containing basal plane defects [16]. However, the solution exfoliation method exhibits high production rates with low or extremely low defect concentration [14].

This work was motivated by the intellectual curiosity to study the most cost-effective method for scalable production of graphene using the solution exfoliation technique and thereby establishing the superiority of this method in comparison to the previously reported methods [22, 23].

\section{Methodology}

The study made use of a domestic kitchen blender, Bajaj GX7 (Bajaj Ltd, India). It comes fitted with a motor of $500 \mathrm{~W}$ which can rotate upto $18000 \mathrm{rpm}$. The impeller is equipped with six blades with a radius of $30 \mathrm{~mm}$ (see Figure 1). Two

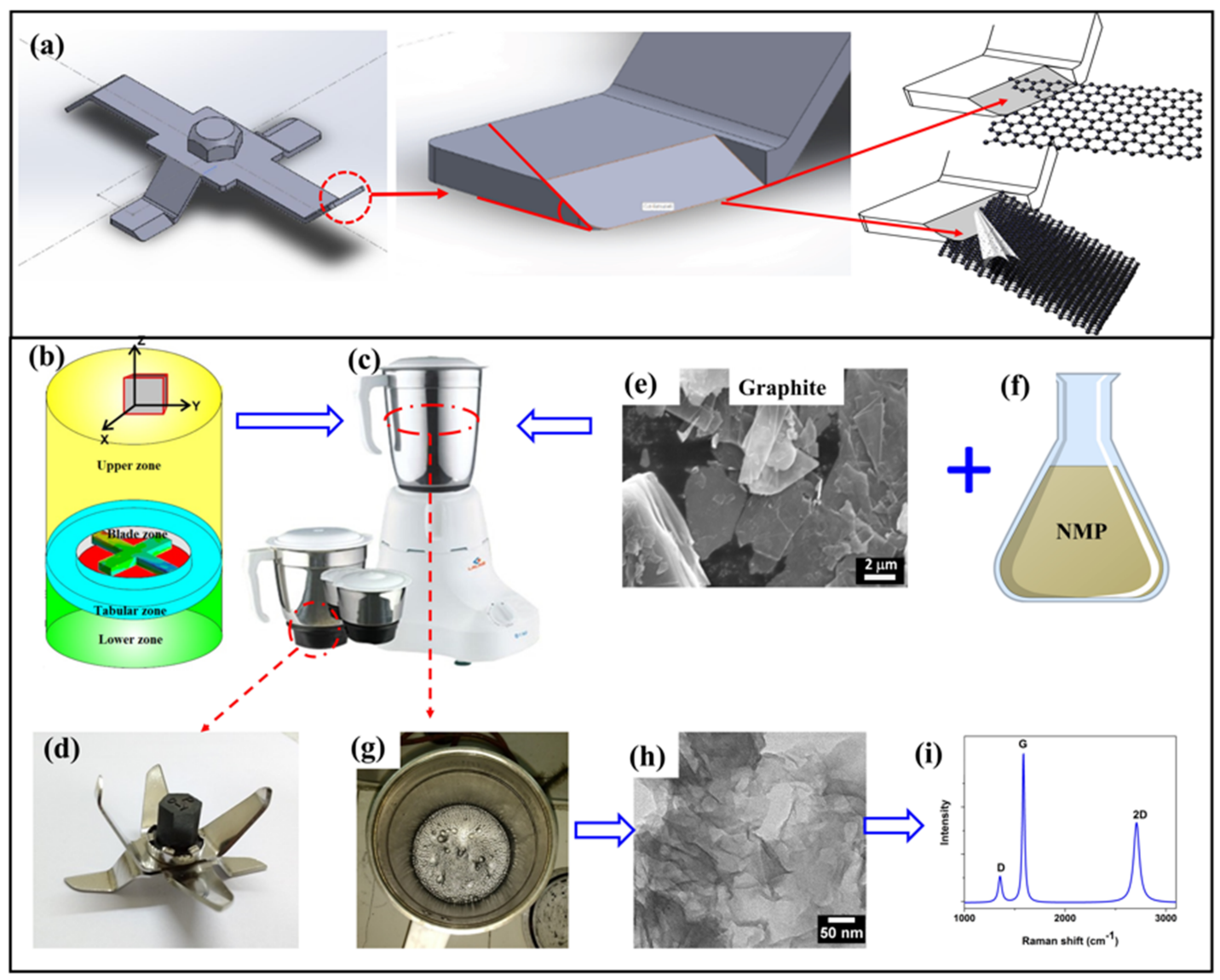

Figure 1: Schematics of graphite exfoliation to graphene in NMP, (a) a scheme of cutting and shear graphene sheet by sharp blade, (b) schematics of the jar, (c) optical image of kitchen blender, (d) grinder blade, (e) graphite flakes (f) NMP (g) prepared graphene in NMP, (h) TEM micrograph of graphene (i) Raman spectrum of graphene. 
blades are angled above the horizontal; two are angled below the horizontal, while the remaining two are almost flat. The radius of the liquidizing jar was $58 \mathrm{~mm}$ (at the bottom), which gradually increases at the top.

The investigation began by dispersing the as-received graphite powder (-100 mesh, 99.9995\%) in N-Methyl-2pyrrolidone (NMP) with an initial concentration of $40 \mathrm{~g} / \mathrm{L}$ in a kitchen blender. $12 \mathrm{~g}$ of graphite powder was taken and mixed with $300 \mathrm{ml} \mathrm{NMP}$ solution in a $1.5 \mathrm{~L}$ jar. The graphite dispersion was treated at different time intervals using a 1 min ON-OFF cycle to avoid over-heating the blender.

Graphene was isolated from the NMP dispersion using four different methods:

(i) Centrifugation (CG): After blending, the grapheneNMP solution was centrifuged twice at $7000 \mathrm{rpm}$ for $15 \mathrm{~min}$. Following centrifugation, the top $80 \%$ of the supernatant was collected and vacuum filtered. Powder deposited on top of the filter paper was collected and dried in vacuum at $70^{\circ} \mathrm{C}$ for 12 hours.

(ii) Sedimentation $(S G)$ : The blended graphene-NMP solution was placed in a beaker and kept for 12 hours for the heavier graphite particles to settle down. Floating graphene was collected using filter paper and repeatedly washed with water to remove NMP. The water-graphene mixture was dried in vacuum oven at $70^{\circ} \mathrm{C}$ for 12 hours.

(iii) Freeze Drying (FDG): After blending, the grapheneNMP solution was kept for further 12 hours. Watergraphene mixture was made, as stated above. Subsequently, graphene was obtained in powder form after freeze-drying.

(iv) Leftover graphene ( $L G$ ): After the blending process, some carbon particles were found stuck onto the wall of the mixing jar (Figure S1). Filling it with water caused graphene to float, and gradually unexfoliated graphite particles settled down. The jar was kept for 12 hours, and after that, floated graphene was collected using a filter paper and washed with water. This water-graphene mixture was vacuum dried at $70^{\circ} \mathrm{C}$ for 12 hours.

For all of the above collection methods, the mixture was mixed homogeneously, and then a portion of the grapheneNMP solution was treated using the above methods. The samples were characterized using Raman spectroscopy (Acton SpectraPro SP-2500, Princeton Instruments, Japan), UVVis spectroscopy (Evolution ${ }^{\mathrm{TM}} 260$ Bio, ThermoFisher Scientific, India), X-ray diffraction (PANalytical), X-ray photoelectron spectroscopy (PHI 5000 Versa Prob II FEI Inc.), Scanning electron microscope (Carl Zeiss EVO 50) and Transmis- sion electron microscope (FEI TECNAI UT-20 operated at $200 \mathrm{kV}$ ).

While the powder samples were directly used for SEM characterization, ultra-pure methanol dispersed graphene was deposited over 400 mesh size formvar/carbon-coated $\mathrm{CU}$ TEM grid and dried overnight for the TEM analysis. Further, the fluoride content absorption test was carried out using a batch experiment in $250 \mathrm{ml}$ Tarson wide-mouthed bottles containing $120 \mathrm{ml}$ of fluoride solution or groundwater. A fixed amount of $0.48 \mathrm{~g}$ of SG was added in each batch $($ solid/solution $=4 \mathrm{~g} / \mathrm{L}$ ). Three batches of each fluoride solution and groundwater were made to check the consistency of the observations. All six batches were kept on a rotatory shaker at $110 \mathrm{rpm}$ for seven days. The volume of 3-5 $\mathrm{ml}$ was collected from the batch at specific time intervals over seven days (12 points) and filtered through $0.2 \mu \mathrm{m}$ disposable syringe filters and collected in a $50 \mathrm{ml}$ Tarson centrifuge tube. Fluoride concentration in the samples was analyzed using Orion 290A F probe.

\section{Results and Discussion}

\subsection{Structural characterization}

The graphene samples were structurally characterized using X-Ray diffraction (XRD), scanning electron microscopy (SEM) and transmission electron microscopy (TEM). The XRD patterns of CG (centrifuged graphene), LG (Leftover graphene) and FDG (Freeze Drying graphene) samples are shown in Figure 2(a).

The XRD patterns confirmed that all types of samples (LG, FDG, CG) collected from the blender were structurally similar in their chemical arrangement. The strongest peak in all the samples corresponds to the (002) peak of graphene. (LG); (FDG); Sedimentation (SG); Centrifugation (CG) Backscattered SEM micrographs of graphite and graphene are shown in Figure 2(b-c), respectively. The TEM bright field image revealed the formation of graphene as shown in Figure 2(d). A clear morphological difference can be observed between graphite and graphene flakes. By comparing the flake sizes of both materials, it can be observed that the size of the graphene (SG) layer is extremely small.

The size of graphite flakes measured using SEM was in the range of $70-80 \mu \mathrm{m}$. Graphite flakes were successfully sheared during liquid-phase exfoliation. A bright field TEM micrograph revealed that the graphene sheets were randomly compacted and stacked; showing uniform laminar morphology such as crumbled silk veil waves. It is evident that the graphene sheets folded and got attached to the grid 

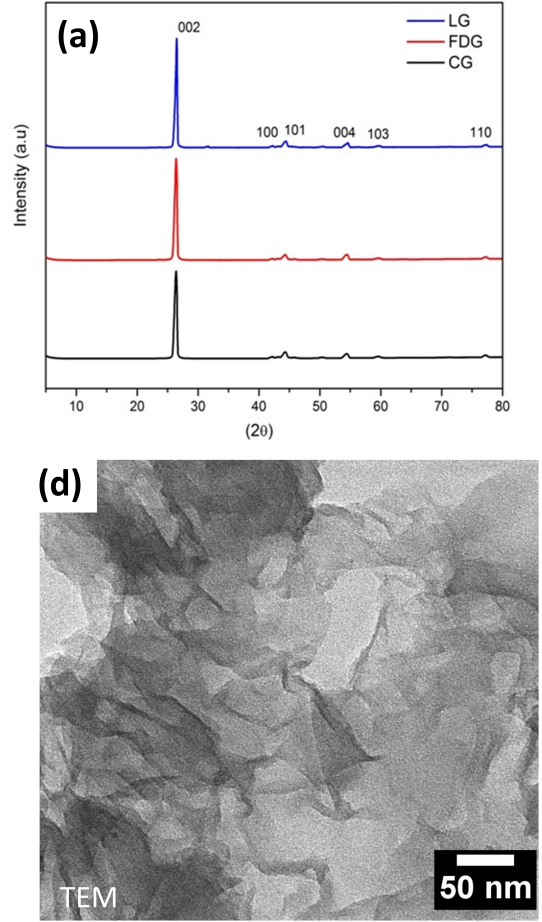
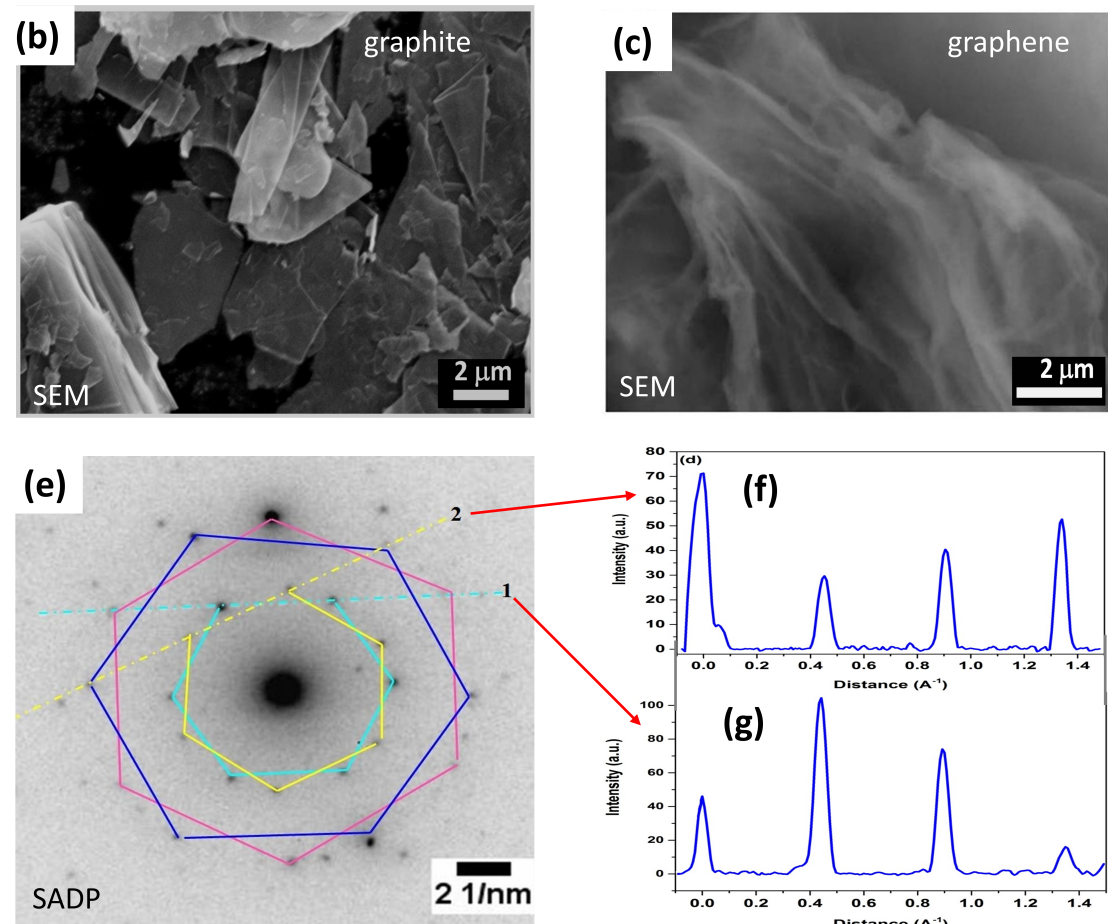

Figure 2: (a) X-ray diffraction pattern of CG, LG and FDG (b) SEM images of the graphite flakes before blending and (c) after blending for 10 minutes, (d) TEM bright-field image of graphene produced after 10 minutes of blending, (e) corresponding SAD pattern; (f) Intensity profile of diffraction spot across line 2 (g) line 1 , intensity profile drawn on the image (e).

only at one side [24]. The selected area diffraction pattern (SADP) corresponding to Figure 2(d) is shown in Figure 2(e). It showed inner and outer hexagons revealing two strong sets of peaks corresponding to tilted planes of graphene. It is well known that the reciprocal space of a single layer crystal consists of a set of rods, arranged as a 2D reciprocal lattice. The measured intensity profile along the $1-1$ and 2-2 (i.e., two rods of the reciprocal space) are shown in Figures 2(f-g). The intensity profile in Figure 2(g) corresponds to single-layer graphene, while Figure 2(f) corresponds to the bi-layer graphene formed during liquid-phase exfoliation of graphite. These distinct points confirmed the crystalline nature of the graphene sheets [24].

Further, XPS measurements were performed on the freeze-dried graphene samples to confirm the graphene quality. C1s and 01s core level spectra of graphene are shown in Figure 3(a-b). A sharp peak at $284.5 \mathrm{eV}$ corresponds to the $s p^{2}$ hybridized carbon, while the peaks at $285.05 \mathrm{eV}, 285.8 \mathrm{eV}$, and $286.75 \mathrm{eV}$ correspond to $\mathrm{C}-\mathrm{H}$ bonds, $\mathrm{C}-\mathrm{N}$ bonds, and $\mathrm{C}=\mathrm{O}$ bonds, respectively [14]. The presence of $\mathrm{O}=\mathrm{C}$ bonds in $\mathrm{O} 1 \mathrm{~s}$ spectra further confirms that these peaks were mainly due to the residual NMP [24]. However, the low intensity of these peaks reveals that NMP was present in very little quantity $[25,26]$.

\subsection{Materials characterisation}

The samples obtained in this work was characterized using Raman spectroscopy. Figure 3(c-e) shows the Raman spectrum of CG. The spectrum reveals the presence of $G$ (graphite), D (defect) and 2D bands as being the signature of graphene. The quality of graphene can be decided by the intensity ratio of $D$ and $G$ peaks, known as. The ratio was always found in the range of 0.03-0.13, which is lower than the values reported earlier [14].

There were two main types of defects observed in graphene: basal plane defects and edge defects. Basal plane defects generally cause broadening of the $\mathrm{G}$ band which was not observed here. However, the weakening D peak signified the presence of edge defects. Moreover, the 2D peak provides a fingerprint signature of graphene [23]. Furthermore, a higher ratio (in the range 0.3-0.6) suggests a better quality of graphene (in terms of the number of layers) as reported earlier [23]. Thus, a lower ratio and a higher ratio indicate the good quality of graphene. Figures 3(d-e) comparing the ratio and ratio for graphenes obtained different blending time after 60 minutes and 70 minutes of blending, respectively highlights the comparative performance. It can be inferred that the centrifuged graphene (CG) and freeze-dried graphene (FDG) were of superior quality as 

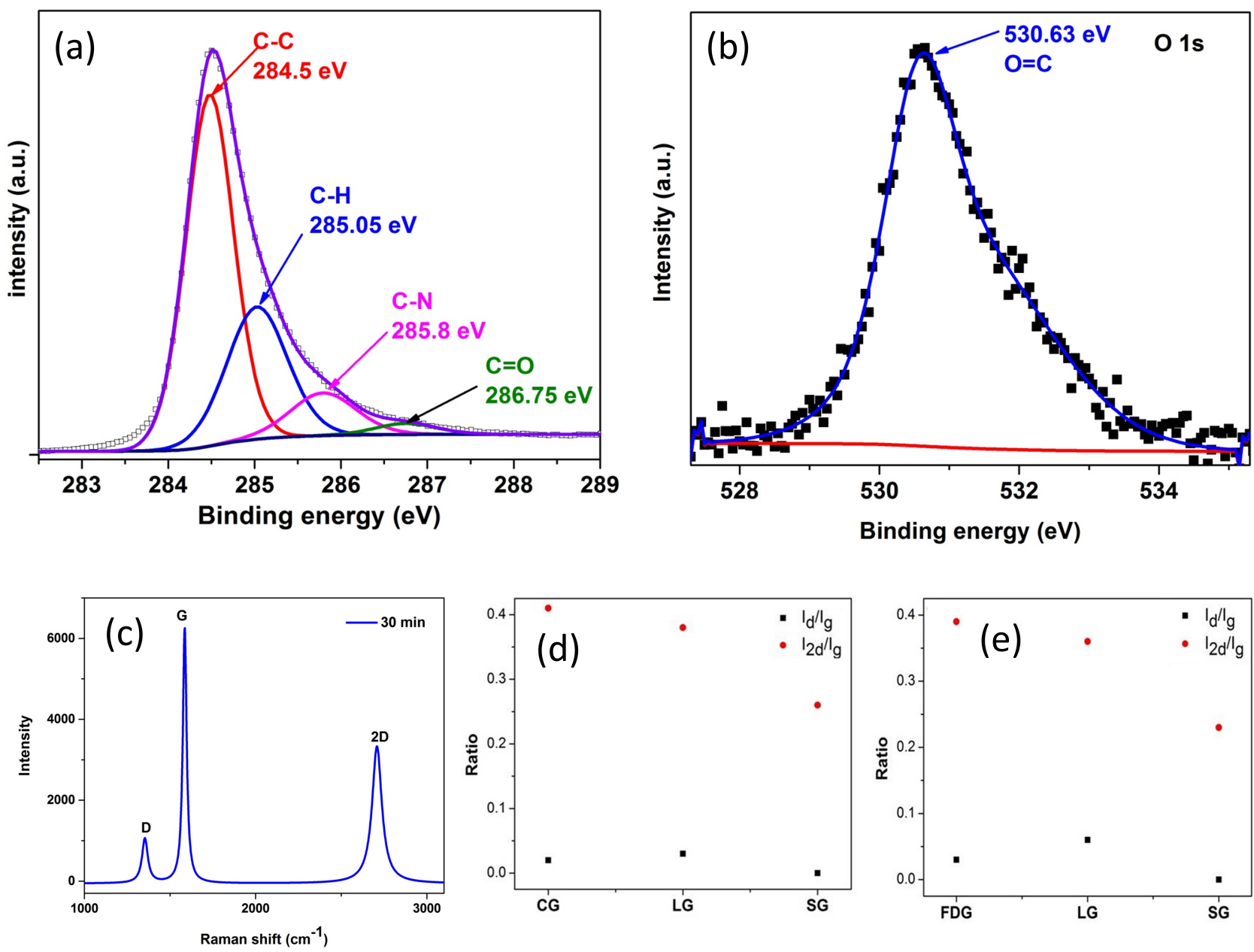

Figure 3: XPS spectra of (a) C1s and (b) 01s of the freeze dried graphene (c) a typical Raman spectrum of CG (centrifuged graphene) after 10 minutes of blending, (d) $I_{d} / I_{g}$ and $I_{2 d} / I_{g}$ ratios obtained from the Raman spectra of FDG, CG, LG and SG blended for 60 minute and (e) 70 minutes (CG-centrifuged graphene; FDG-freeze-dried graphene; LG-leftover graphene; SG-sedimentation graphene).

compared to LG and SG. During exfoliation of graphene, bubble formation was observed to enhance the quality of graphene and yield (detailed in supporting information). Figure 4(a) showed typical UV-Vis spectra obtained from CG graphene sample. It reveals an intense peak at $271 \mathrm{~nm}$ corresponding to $\mathrm{p}^{\circledR} \mathrm{p}^{*}$ electronic transition of $\mathrm{C}=\mathrm{C}$ bond of graphene. Furthermore, the concentration of graphene dispersion was also calculated from the UV-Vis spectra according to Beer-Lambert law as shown in Figure 4(b). The concentration of graphene was found to increase continually with time. Typically it was about $25.5 \mu \mathrm{g} / \mathrm{ml}$ after 10 minutes of exfoliation. The yield obtained during a simple trial was $\sim 0.30 \mathrm{~g} / \mathrm{h}$ and with further optimisation, the method can certainly be improved further.

\subsection{Fluoride ions/color absorption}

The fluoride ions absorption was performed on fluorinated water and synthetic groundwater. The fluorinated water consisted of ultrapure water mixed with $\mathrm{NaF}$ stock solution to provide the appropriate fluoride in the solution. The amount of fluoride was kept the same as in the synthetic groundwater and was set at $7.98 \mathrm{ppm}$. The $7.98 \mathrm{ppm}$ was chosen because the contaminated groundwater has fluoride present in this much concentration.

The primary purpose of using synthetic groundwater is to study the adsorption of fluoride in the presence of competing anions $\left(\mathrm{Mg}^{++}, \mathrm{K}^{+}, \mathrm{Ca}^{++}, \mathrm{Na}^{+}, \mathrm{Cl}^{-}\right)$. The two solutions were used to compare the fluoride uptake capacity of centrifuged graphene (SG). Most of the studies to date have generally reported the behavior of adsorbents in the absence of other competing ions present in the groundwater requiring treatment. Figure 4(c) shows the change in fluoride con- 

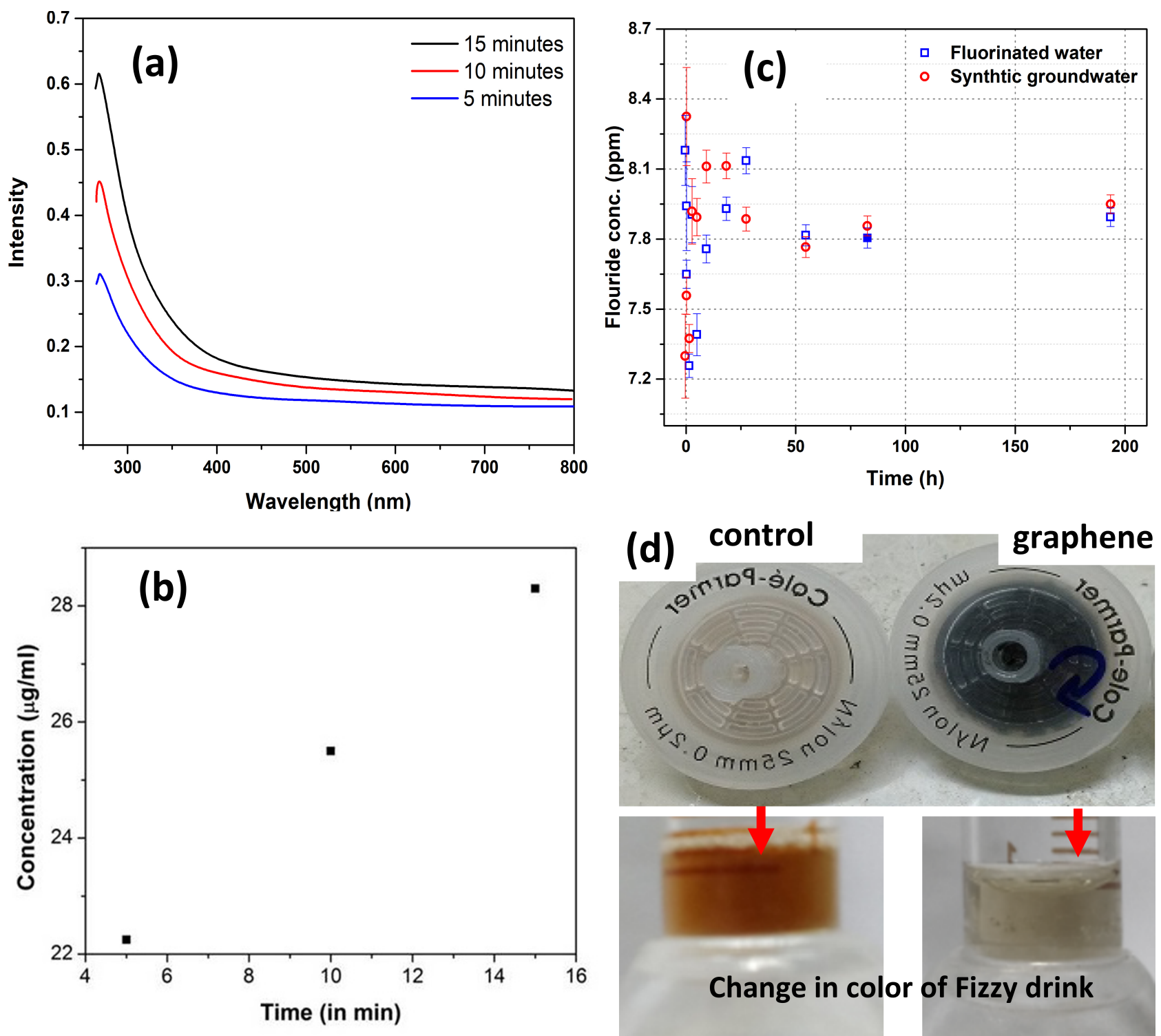

Figure 4: (a) UV-Vis absorption spetra of CG for a different time duration of blending, (b) concentration of graphene produced calculated using Beer-Lambert law, (c) Fluoride uptake on graphene in synthetic groundwater and fluoride solution (d) change in color of general fizzy drink passing through normal filter and prepared graphene deposited filter.

centration (in ppm) with time (in hours). Initial fluoride concentration in both fluoride and synthetic groundwater was $7.98 \mathrm{ppm}$. Initially, there is a slight increase in the fluoride concentration, which is unexpected. The only explanation for this observation could be the interference of the probe due to other types of ions. After 10 minutes of an initial increase, fluoride concentration continuously goes down till 1 hour to $7.3 \mathrm{ppm}$ in fluoride and synthetic groundwater. However, the fluoride concentration again increases, which might be due to the oversaturation of graphene. The maximum decrease of fluoride was observed to be $7.3 \mathrm{ppm}$ or about $\sim 10 \%$ decrease in fluoride concentration. Simi- larly, a qualitative test was performed on fizzy drink, which was passed through graphene deposited filter and normal filter. An excellent color absorption ability of graphene was observed while passing the drink through the graphene deposited filter compared to a normal filter and this is also shown in Figure 4(d). 


\section{Conclusions}

The present work reports a sustainable nanofabrication route for the large scale manufacture of defect-free graphene based on the principle of mechanical exfoliation by a simple kitchen blender. The method was seen to be transformative for fabricating graphene for a wide range of applications such as catalysis, sensing, nanocomposites and functional decoration with different kinds of nanoparticles. The propensity of exfoliation was observed to enhance by the turbulent flow conditions in presence of a liquid solvent in the blender. The graphene produced by this method when assessed using Raman spectroscopy was observed to have an $\mathrm{I}_{d} / \mathrm{I}_{g}$ ratio below 0.1 and $\mathrm{I}_{2 d} / \mathrm{I}_{g}$ ratio less than 0.45 as well as a yield of $0.30 \mathrm{~g} / \mathrm{h}$. These values highlight the superiority of the investigated method to manufacture graphene over previously reported exfoliation methods. Increasing the duration of blending was observed to cause an increase in the yield of graphene. Moreover, the centrifuged separated graphene was observed to remove $\sim 10 \%$ fluoride ions from groundwater and was observed to be capable to absorb colors from the solvent/fizzy drinks.

Funding information: All authors greatly acknowledge the financial support provided by SERB-DST India. SG greatly acknowledge the financial support provided by the UKRI via Grants No. EP/L016567/1, EP/S013652/1, EP/S036180/1, EP/T001100/1 and EP/T024607/1, TFIN+ Feasibility study award to LSBU (EP/V026402/1), the Royal Academy of Engineering via Grants No. IAPP18-19\295 and TSP1332, EURAMET EMPIR A185 (2018), the EU Cost Action (CA15102, CA18125, CA18224 and CA16235) and the Newton Fellowship award from the Royal Society (NIF $\backslash R 1 \backslash 191571)$. Wherever applicable, the work made use of Isambard Bristol, UK supercomputing service accessed by a Resource Allocation Panel (RAP) grant as well as ARCHER resources (Project e648).

Conflict of interest: The authors declare no conflict of interest.

Ethical approval: The conducted research is not related to either human or animal use

Data availability statement: Data sharing is not applicable

\section{References}

[1] Yu X, Cheng H, Zhang M, Zhao Y, Qu L, Shi G. Graphene-based smart materials. Nat Rev Mater. 2017;2(9):17046.

[2] Liu M, Weston PJ, Hurt RH. Controlling nanochannel orientation and dimensions in graphene-based nanofluidic membranes. Nat Commun. 2021 Jan;12(1):507.

[3] Khan S, Achazhiyath Edathil A, Banat F. Sustainable synthesis of graphene-based adsorbent using date syrup. Sci Rep. 2019 Dec;9(1):18106.

[4] Andrei EY, MacDonald AH. Graphene bilayers with a twist. Nat Mater. 2020 Dec;19(12):1265-75.

[5] Wang L, Sofer Z, Pumera M. Will Any Crap We Put into Graphene Increase Its Electrocatalytic Effect? ACS Nano. 2020 Jan;14(1):215.

[6] Akinwande D, Huyghebaert C, Wang CH, Serna MI, Goossens S, Li LJ, et al. Graphene and two-dimensional materials for silicon technology. Nature. 2019 Sep;573(7775):507-18.

[7] Romagnoli M, Sorianello V, Midrio M, Koppens FH, Huyghebaert C, Neumaier D, et al. Graphene-based integrated photonics for next-generation datacom and telecom. Nat Rev Mater. 2018;3(10):392-414.

[8] Bhuyan MS, Uddin MN, Islam MM, Bipasha FA, Hossain SS. Synthesis of graphene. Int Nano Lett. 2016;6(2):65-83.

[9] Bueno RA, Martínez JI, Luccas RF, Del Árbol NR, Munuera C, Palacio I, et al. Highly selective covalent organic functionalization of epitaxial graphene. Nat Commun. 2017 May;8(1):15306.

[10] Vecera P, Chacón-Torres JC, Pichler T, Reich S, Soni HR, Görling A, et al. Precise determination of graphene functionalization by in situ Raman spectroscopy. Nat Commun. 2017 May;8(1):15192.

[11] Rius G, Perez-Murano F, Yoshimura M. Graphene crystal growth by thermal precipitation of focused ion beam induced deposition of carbon precursor via patterned-iron thin layers. Nanofabrication. 2014;1(1).

[12] Thakur S, Verma A, Alsanie WF, Christie G, Thakur VK. On the graphene and its derivative based polymer nanocomposites for glucose sensing. Mater Lett. 2022;307:130971.

[13] Sharma B, Thakur S, Trache D, Yazdani Nezhad H, Thakur VK. Microwave-Assisted Rapid Synthesis of Reduced Graphene Oxide-Based Gum Tragacanth Hydrogel Nanocomposite for Heavy Metal Ions Adsorption. 2020;10(8):1616.

[14] Paton KR, Varrla E, Backes C, Smith RJ, Khan U, O’Neill A, et al. Scalable production of large quantities of defect-free fewlayer graphene by shear exfoliation in liquids. Nat Mater. 2014 Jun;13(6):624-30.

[15] Kosynkin DV, Higginbotham AL, Sinitskii A, Lomeda JR, Dimiev A, Price BK, et al. Longitudinal unzipping of carbon nanotubes to form graphene nanoribbons. Nature. 2009 Apr;458(7240):8726.

[16] Marcano DC, Kosynkin DV, Berlin JM, Sinitskii A, Sun Z, Slesarev A, et al. Improved synthesis of graphene oxide. ACS Nano. 2010 Aug;4(8):4806-14.

[17] Devi MM, Sahu SR, Mukherjee P, Sen P, Biswas K. Graphene: a self-reducing template for synthesis of graphene-nanoparticles hybrids. RSC Advances. 2015;5(76):62284-9.

[18] Choucair M, Thordarson P, Stride JA. Gram-scale production of graphene based on solvothermal synthesis and sonication. Nat Nanotechnol. 2009 Jan;4(1):30-3. 
[19] Wu ZS, Ren W, Gao L, Zhao J, Chen Z, Liu B, et al. Synthesis of graphene sheets with high electrical conductivity and good thermal stability by hydrogen arc discharge exfoliation. ACS Nano. 2009 Feb;3(2):411-7.

[20] Mattevi C, Kim H, Chhowalla M. A review of chemical vapour deposition of graphene on copper. J Mater Chem. 2011;21(10):332434.

[21] Paton KR, Anderson J, Pollard AJ, Sainsbury T. Production of few-layer graphene by microfluidization. Mater Res Express. 2017;4(2):025604.

[22] Varrla E, Paton KR, Backes C, Harvey A, Smith RJ, McCauley J, et al. Turbulence-assisted shear exfoliation of graphene using household detergent and a kitchen blender. Nanoscale. 2014 Oct;6(20):11810-9.
[23] Yi M, Shen Z. Kitchen blender for producing high-quality fewlayer graphene. Carbon. 2014;78:622-6.

[24] Meyer JC, Geim AK, Katsnelson MI, Novoselov KS, Obergfell D, Roth S, et al. On the roughness of single- and bi-layer graphene membranes. Solid State Commun. 2007;143(1):101-9.

[25] Gupta A, Chen G, Joshi P, Tadigadapa S, Eklund PC. Raman scattering from high-frequency phonons in supported n-graphene layer films. Nano Lett. 2006 Dec;6(12):2667-73.

[26] Zhang L, Yu J, Yang M, Xie Q, Peng H, Liu Z. Janus graphene from asymmetric two-dimensional chemistry. Nat Commun. 2013;4(1):1443. 